\title{
Hyponatremia as a Predictor of Adverse Outcome in Children With Severe Lower Respiratory Tract Infection in Tribhuvan University Teaching Hospital (Tuth), Nepal
}

\section{CURRENT STATUS: POSTED}

5 Research Square

\section{Machhindra Lamichhane}

Dhaulagiri Zonal Hospital

archanamachhindra2045@gmail.comCorresponding Author

Laxman Shrestha

Institute of medicine, Maharajgunj Medical Campus

Luna Bajracharya

Maharajgunj Mediacl Campus, Kathmandu

Archana Bagale

Yeti Health Science Academy

DOI:

10.21203/rs.2.20441/v1

\section{SUBJECT AREAS}

\section{Pediatrics Pulmonology}

KEYWORDS

Hyponatremia, Severe LRTI, TUTH 
Abstract

Introduction: Lower respiratory tract infection (LRTI) is one of the major causes of mortality in children with estimated 1 million deaths every year. Hyponatremia is the most common electrolyte abnormality seen in LRTI.

Objective: To find association of hyponatremia (serum sodium $<135 \mathrm{meq} /$ ) with severe LRTI. Design: Prospective cross sectional study

Settings: Pediatric Emergency, Ward and Pediatric Intensive Care Unit of Tribhuvan University Teaching Hospital(TUTH)

Subjects: Children between 2 months to 16 years presenting with cough for less than 3 weeks, fast breathing and chest indrawing.

Methods: Children between 2 month to 16 years of age having cough, fast breathing and chest indrawing admitted in emergency, ward or PICU of TUTH were screened and among them, children having pediatric respiratory severity score of 4-5 were enrolled in the study. Serum sodium was sent during admission and collected within 2 hours. Daily follow up was done to find need of respiratory support, duration to resolve hypoxia, total duration in hospital and final outcome of patient. Statistical test applied were Chi-square test and Fisher exact test.

Results: $47.5 \%$ of study population had hyponatremia, of whom $65.79 \%$ had mild hyponatremia $26.31 \%$ had moderate and $7.89 \%$ had severe hyponatremia. Association of hyponatremia with need of non rebreathing face mask $(p=0.001)$, ventilatio $r(p=0.009)$, duration of hospital stay $(p=0.047)$ were significant. The study also found the association of severity of hyponatremia with need of non rebreathing facemask $(p=0.001)$, ventilator $(p=0.01)$, outcome $(p=0.001)$, duration of stay $(0.002)$. Mean time to resolve hypoxia in children with hyponatremia (4.5 days) was longer compared to normal sodium (2.58 days). Four patients died during the study period and all of them had hyponatremia.

Conclusion- Association of hyponaremia and severity of hyponatremia in severe LRTI with need of non-rebreathing face mask, ventilatior, outcome, duration of stay was significant. Full Text 
Due to technical limitations, full-text HTML conversion of this manuscript could not be completed.

However, the manuscript can be downloaded and accessed as a PDF.

Figures

Meeting inclusion criteria and screened ( $\mathrm{n}=90$ )

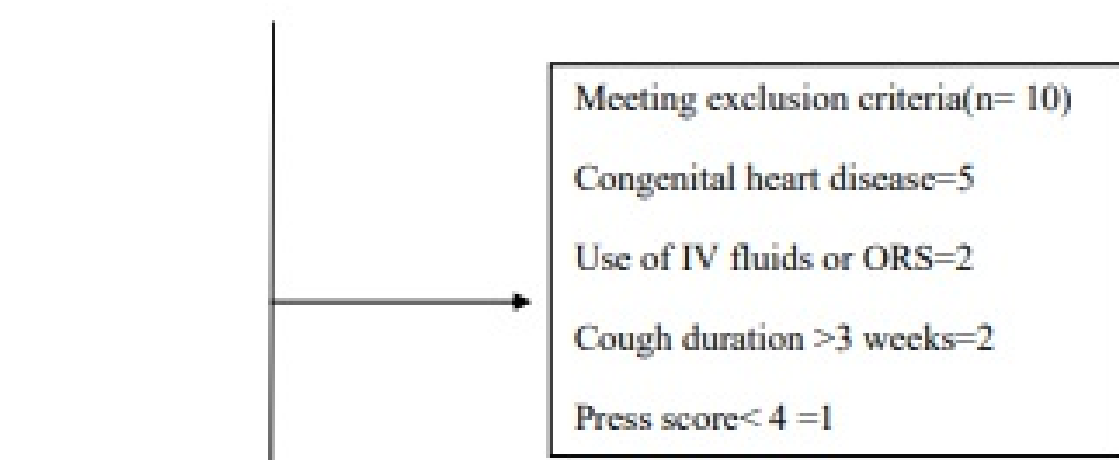

Completed study $(\mathrm{n}=80)$

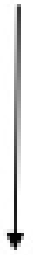

Available for final analysis $(\mathrm{n}=80)$

Figure 1

Flow chart of screened and enrolled cases in the study 


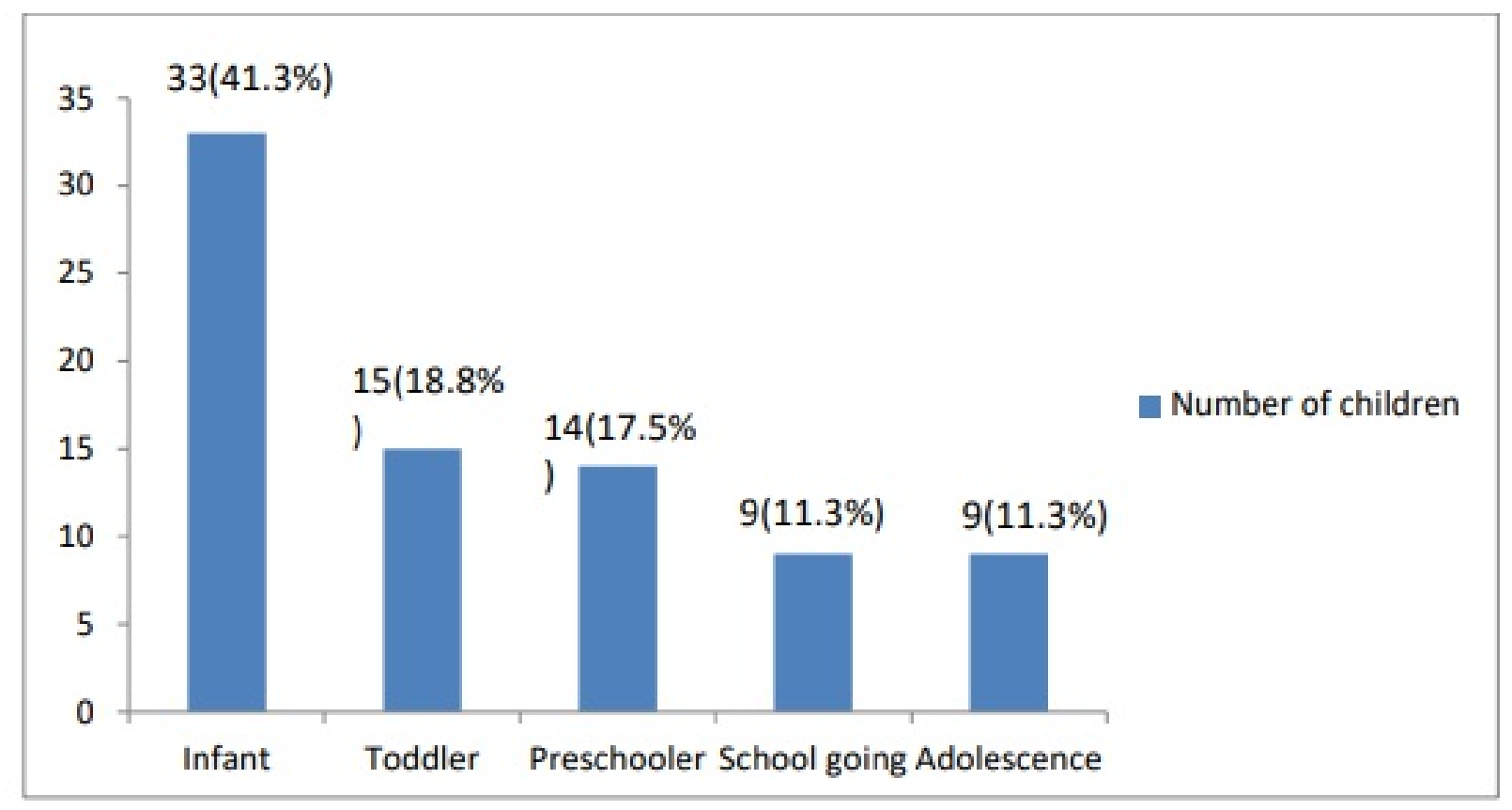

Figure 2

Age of study population

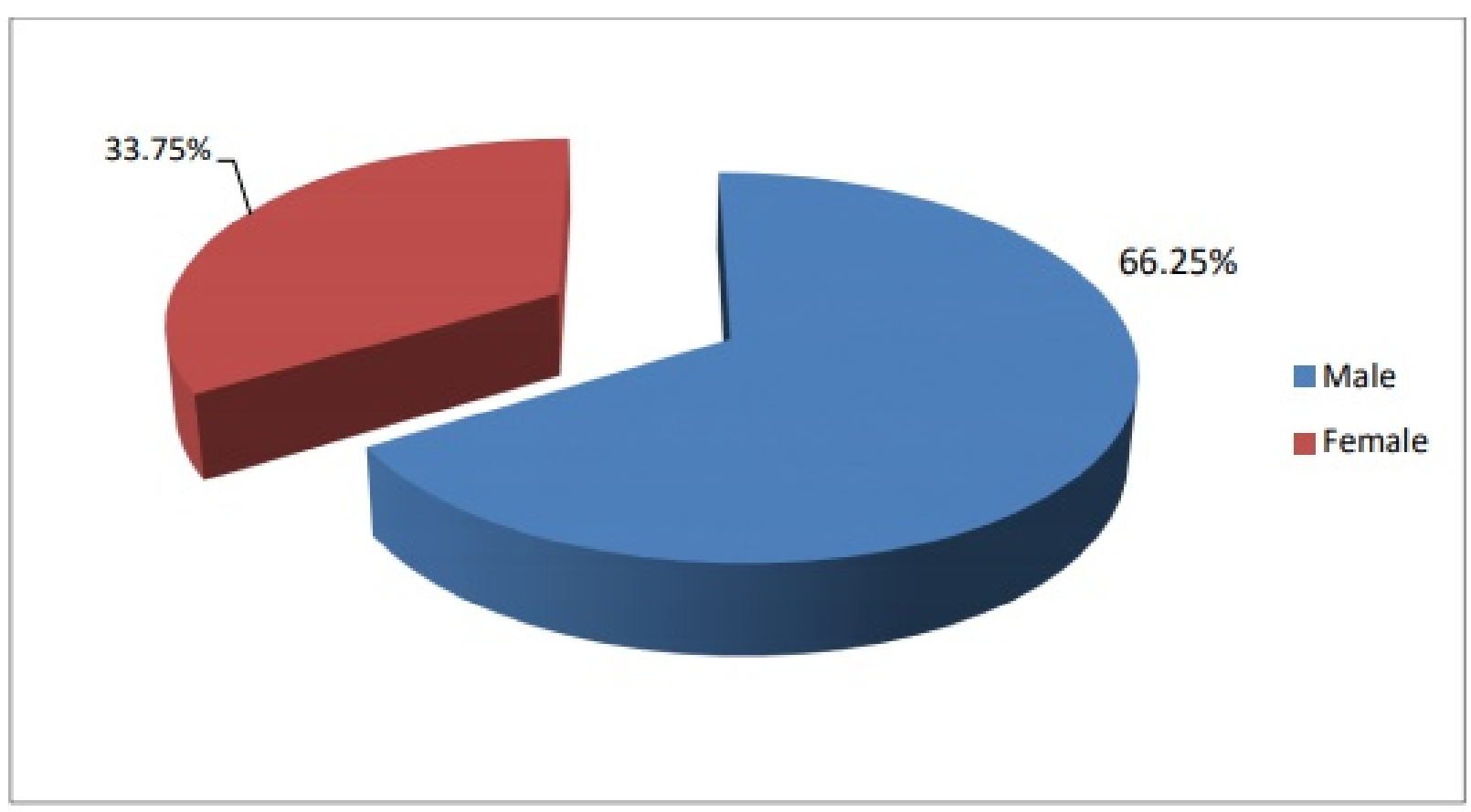

Figure 3

Sex of study population

\section{Supplementary Files}

This is a list of supplementary files associated with this preprint. Click to download. 
Data.xls

Decleration.pdf 